\title{
LMI-BASED ADAPTIVE FUZZY INTEGRAL SLIDING MODE CONTROL OF MISMATCHED UNCERTAIN SYSTEMS
}

\author{
CHAOUKI MNASRI, MONCEF GASMI
}

\author{
Research Unit in Automatic Control and Industrial Computing, URAII \\ National Institute of Applied Sciences and Technology, INSAT \\ Carthage University, Zone urbaine Nord, BP 676, Tunis Cedex, 1080, Tunisia \\ e-mail: chaouki.mnasriaisetgf.rnu.tn, m.gsmeenit.rnu.tn
}

\begin{abstract}
Integral sliding mode design is considered for a class of uncertain systems in the presence of mismatched uncertainties in both state and input matrices, as well as norm-bounded nonlinearities and external disturbances. A sufficient condition for the robust stability of the sliding manifold is derived by means of linear matrix inequalities. The initial existence of the sliding mode is guaranteed by the proposed control law. The improvement of the proposed control scheme performances, such as chattering elimination and estimation of norm bounds of uncertainties, is then considered with the application of an adaptive fuzzy integral sliding mode control law. The validity and efficiency of the proposed approaches are investigated through a sixth order uncertain mechanical system.
\end{abstract}

Keywords: adaptive fuzzy control, integral sliding mode, LMI, mismatched uncertainties.

\section{Introduction}

The relationship between models and the reality they represent is subtle and complex. The differences or errors between them define the term uncertainty. Thus, it is necessary to analyze dynamic systems subject to uncertainties. In other words, for control design purposes we need to handle simple models. However, the obtained controller must work when connected to a real system. Control strategies based on this philosophy have attracted the attention of researchers and have been widely considered. Variable Structure Control (VSC) with Sliding Modes (SMs) has been regarded as a robust technique for its insensitivity to external disturbances and model uncertainties satisfying the matching condition, i.e., perturbations that affect the system model through the input channel (Decarlo et al., 1988; Hung et al., 1993). In addition, the use of SMC can offer fast response, good transient performance and order reduction. These advantages make the SM technique very widespread in robust control design (Ha et al., 1999; Utkin, 1992).

The existence of the reaching phase in the resulting closed loop system yields the sensitivity of the dynamics to perturbations in an initial period of time in which the system has not yet reached the sliding manifold (Ackermann and Utkin, 1998). Hence, the new approach known as Integral SMC (ISMC) has as the main contribution the elimination of the reaching phase. The basic idea of this concept is the addition of an integral term in the sliding surface allowing immediate sliding mode emergence (Utkin and Shi, 1996). ISMC is used in the works of Mnasri and Gasmi (2007a; 2007b) and good results are obtained for large scale systems with matched uncertainties.

Application of the same method in the control of matched uncertain MIMO systems produced good performance as for robustness and tracking (Mnasri and Gasmi, 2008). However, in many cases, the uncertainties and external disturbances do not always satisfy the matching conditions. Contrary to the matched case, any mismatched uncertainty affects the behavior of the sliding mode directly, even when the ISMC approach is used. To solve this problem, the main idea is the combination of SMC with other robust techniques.

A majority of the existing methods are based on Classic SMC (CSMC) (Kim et al., 2000; Choi, 2001; Xia and Jia, 2003). These methods are affected by the aforementioned insufficiency of SMC with the reaching phase. Recently, much research has been focused on the advantages of ISMC in the control of systems with mismatched uncertainties. This method is considered by $\mathrm{Cao}$ and $\mathrm{Xu}$ (2004) for the case of systems with mismatched uncer- 
tainties in the state matrix, but it is limited to matched uncertainties in the input matrix and to external disturbances. A few recent studies have included the case of mismatched uncertainties in the input matrix (Shaocheng and Yongji, 2006; Choi, 2007). However, neither of them is applicable in the presence of mismatched disturbances not related to the input channel.

In this paper, we propose a new approach to design ISMC for a class of uncertain systems. This class regroups mismatched uncertainties in both state and input matrices, as well as mismatched norm-bounded nonlinearities and external disturbances. Our approach, based on Linear Matrix Inequalities (LMIs), combines the advantages of ISMC with $H_{\infty}$ control. This gives a sufficient condition for the robust stability of the system in the sliding mode. The chattering phenomenon, the main drawback of SMC, is also considered in this paper. An adaptive fuzzy ISMC law is proposed to improve the performance of the control scheme, by allowing elimination of chattering and the estimation of the norm bounds of uncertainties. The efficiency of the proposed control laws is investigated through a sixth-order mechanical system example.

\section{Problem formulation}

2.1. System description. Consider the following uncertain system:

$$
\left\{\begin{aligned}
\dot{x}= & {[A+\Delta A] x } \\
& +[B+\Delta B] u(t)+f(x, t)+H \omega(t), \\
y= & C x
\end{aligned}\right.
$$

where $x \in \mathbb{R}^{n}$ is the state vector, $u \in \mathbb{R}^{m}$ is the input control, $y \in \mathbb{R}^{q}$ is the controlled output, $f(x, t) \in \mathbb{R}^{n}$ is the vector of nonlinearities and unmodelled dynamics. Here $\omega \in \mathbb{R}^{p}$ is a square-integrable external disturbance. $A \in \mathbb{R}^{n \times n}$ is the system characteristic matrix, $B \in \mathbb{R}^{n \times m}$ is the input matrix, $H \in \mathbb{R}^{n \times p}$ is the matrix of external disturbances, $C \in \mathbb{R}^{q \times n}$ is the output matrix, $\Delta A$ and $\Delta B$ represent system and input matrix uncertainties, respectively. We make the following assumptions:

(i) The pair $(A, B)$ is stabilizable.

(ii) The input matrix $B$ has full rank.

(iii) There exist known positive constants $a, b, g$, and $\omega_{0}$ such that $\|\Delta A\| \leq a,\|\Delta B\| \leq b,\|f(x, t)\| \leq g\|x\|$ and $\|\omega(t)\| \leq \omega_{0}$, for all $t \in \mathbb{R}^{+}$.

(iv) $\left\|B^{+} \Delta B\right\| \leq b_{m}<1$, where $b_{m}$ is a positive known scalar and $B^{+} \equiv\left(B^{T} B\right)^{-1} B^{T}$.

2.2. Preliminary results. In this section, we give some preliminary results that will be helpful to obtain our main results.
Lemma 1. (Boyd et al., 1994) Consider the following unforced system:

$$
\left\{\begin{array}{l}
\dot{x}=A x+H \omega \\
y=C x
\end{array}\right.
$$

This system is quadratically stable and satisfies the $H_{\infty}$ constraint $\left\|T_{y \omega}\right\|_{\infty}<\gamma$ if there exists a quadratic Lyapunov function $V(x)=x^{T} P x, P>0$ such that, for all $t>0$,

$$
\dot{V}+y^{T} y-\gamma^{2} \omega^{T} \omega<0 .
$$

Lemma 2. (Choi, 2007) For any vectors $x$ and $y$ with appropriate dimensions, the following inequality holds:

$$
2 x^{T} y \leq \epsilon x^{T} x+\epsilon^{-1} y^{T} y, \quad \forall \epsilon>0 .
$$

Lemma 3. (Boyd et al., 1994) Consider a block symmetric matrix

$$
\left[\begin{array}{cc}
A & B^{T} \\
B & C
\end{array}\right]
$$

where $A$ and $C$ are square matrices, with $C$ being negative definite. This matrix is negative definite if and only if $A-B^{T} C^{-1} B$ is negative semi-definite.

\section{Sliding mode stability}

3.1. Sliding surface choice. In this work, we choose the switching function as follows:

$$
S(t)=B^{+} x+z,
$$

where $z \in \mathbb{R}^{m}$ is the solution of the following dynamic equation:

$$
\dot{z}=-\left(B^{+} A+K\right) x, z(0)=-B^{+} x(0),
$$

where $K \in \mathbb{R}^{m \times n}$ is a state feedback gain which should be designed to lead the closed loop system to the desired performances in the sliding mode. The sliding surface considered allows the elimination of the reaching phase characterizing CSMC, because the initial value $S(0)=0$ for any initial conditions. The time derivative of the switching function is derived using (1) and (6) as follows:

$$
\begin{aligned}
\dot{S}(t)= & B^{+}[[A+\Delta A] x+[B+\Delta B] u+f+H \omega] \\
& -B^{+} A x-K x \\
= & B^{+}[\Delta A x+[B+\Delta B] u+f+H \omega]-K x .
\end{aligned}
$$

Let us suppose that

$$
\Gamma=I_{n}-B B^{+},
$$


where $I_{n} \in \mathbb{R}^{n \times n}$ is the $n \times n$ identity matrix. Accordingly, it is easy to deduce that

$$
B^{+} \Gamma=B^{+}-B^{+} B B^{+}=B^{+}-B^{+}=0 .
$$

In addition, we can rewrite the uncertainty terms as follows:

$$
\begin{aligned}
\Delta A(t) & =B \Delta A_{m}(t)+\Delta A_{u}(t), \\
\Delta B(t) & =B \Delta B_{m}(t)+\Delta B_{u}(t), \\
f(x, t) & =B f_{m}(x, t)+f_{u}(x, t), \\
H & =B H_{m}+H_{u},
\end{aligned}
$$

where

$$
\begin{aligned}
\Delta A_{m}(t) & =B^{+} \Delta A(t), & \Delta B_{m}(t) & =B^{+} \Delta B(t), \\
f_{m}(x, t) & =B^{+} f(x, t), & H_{m} & =B^{+} H, \\
\Delta A_{u}(t) & =\Gamma \Delta A(t), & \Delta B_{u}(t) & =\Gamma \Delta B(t), \\
f_{u}(x, t) & =\Gamma f(x, t), & H_{u} & =\Gamma H .
\end{aligned}
$$

Furthermore, there exist known positive constants $a_{m}, a_{u}$, $b_{u}, g_{m}$, and $g_{u}$ such that $\left\|\Delta A_{m}\right\| \leq a_{m},\left\|\Delta A_{u}\right\| \leq a_{u}$, $\left\|\Delta B_{u}\right\| \leq b_{u},\left\|f_{m}(x, t)\right\| \leq g_{m}\|x\|$, and $\left\|f_{u}(x, t)\right\| \leq$ $g_{u}\|x\|$.

Therefore, using (9)-(12), Eqn. (8) can be transformed into

$$
\begin{aligned}
\dot{S}= & \Delta A_{m} x+\left(I_{m}+\Delta B_{m}\right) u \\
& +f_{m}(x, t)+H_{m} \omega-K x .
\end{aligned}
$$

The intrinsic condition of the sliding mode emergence is

$$
S(t)=0, \quad \dot{S}(t)=0 .
$$

This condition allows deriving the expression for the equivalent control as follows:

$$
\begin{aligned}
u_{e q}=- & \left(I_{m}+\Delta B_{m}\right)^{-1} \\
& \times\left[\Delta A_{m} x+f_{m}(x, t)+H_{m} \omega-K x\right] .
\end{aligned}
$$

Remark 1. Equation (15) requires that the matrix $\left(I_{m}+\right.$ $\left.\Delta B_{m}\right)$ be nonsingular. This requirement is guaranteed by Assumption (iv).

3.2. Stability of the sliding motion. By substituting (15) in (1), the sliding mode dynamics can be described by

$$
\begin{aligned}
\dot{x}(t)= & A x+B K x+\tilde{B} K x+\Delta A_{u} x-\tilde{B} \Delta A_{m} x \\
& +f_{u}-\tilde{B} f_{m}+H_{u} \omega-\tilde{B} H_{m} \omega,
\end{aligned}
$$

where

$$
\tilde{B}=\Delta B_{u}\left(I_{m}+\Delta B_{m}\right)^{-1} .
$$

It is clear from (16) that the system dynamics in the sliding mode are affected by the existence of uncertainties and disturbances. Our objective is the design of a state feedback gain $K$. This gain guarantees the stability of the closed loop system while satisfying the $H_{\infty}$ constraint $\left\|T_{y \omega}\right\|_{\infty}<\gamma$. In order to reach this goal, we proceed by means of the LMI method.

Theorem 1. The uncertain system (1) with the assumptions (i)-(iv) is quadratically stable on the sliding surface described by (6) and satisfies the $H_{\infty}$ constraint $\left\|T_{y \omega}\right\|_{\infty}<\gamma$ if there exist a symmetric positivedefinite matrix $X$, a matrix $R$ and positives scalars $\epsilon_{i}, i=$ $1, \ldots, 6$, such that the following LMI holds:

$$
\left[\begin{array}{ll}
\Xi & \Theta \\
* & \Psi
\end{array}\right]<0
$$

where

$$
\begin{aligned}
\Xi & =\left[\begin{array}{ccccc}
\Sigma & X C^{T} & a_{u} X & b_{u} a_{m} X & g_{u} X \\
* & -I & 0 & 0 & 0 \\
* & * & -\epsilon_{1} I & 0 & 0 \\
* & * & * & -\epsilon_{2}^{*} I & 0 \\
* & * & * & * & -\epsilon_{3} I
\end{array}\right], \\
\Sigma & =A X+X A^{T}+B R+R^{T} B^{T}+\sum_{i=1}^{6} \epsilon_{i} I, \\
R & =K X, \quad \epsilon_{i}^{*}=\left(1-b_{m}\right)^{2} \epsilon_{i}, \\
\Theta & =\left[\begin{array}{cccc}
b_{u} g_{m} X & b_{u} R^{T} & H_{u} & 0 \\
0 & 0 & 0 & 0 \\
0 & 0 & 0 & 0 \\
0 & 0 & 0 & 0 \\
0 & 0 & 0 & 0
\end{array}\right], \\
\Psi & =\left[\begin{array}{cccc}
-\epsilon_{4}^{*} I & 0 & 0 & 0 \\
* & -\epsilon_{5}^{*} I & 0 & 0 \\
* & * & -\gamma^{2} I & b_{u} H_{m}^{T} \\
* & * & * & -\epsilon_{6}^{*} I
\end{array}\right] .
\end{aligned}
$$

Here the asterisk denotes the transpose of the corresponding block above the main diagonal, and I denotes the identity matrix of appropriate dimension.

Proof. Consider a symmetric positive-definite matrix $P$ and choose a candidate Lyapunov function,

$$
V(x)=x^{T} P x .
$$

In order to complete the proof, we proceed by verification of Lemma 1:

$$
\begin{aligned}
\dot{V}+ & y^{T} y-\gamma^{2} \omega^{T} \omega \\
= & x^{T}\left[P A+A^{T} P+P B K+K^{T} B^{T} P\right] x \\
& +2 x^{T} P \Delta A_{u} x-2 x^{T} P \tilde{B} \Delta A_{m} x+2 x^{T} P f_{u} \\
& -2 x^{T} P \tilde{B} f_{m}+2 x^{T} P \tilde{B} K x+2 x^{T} P H_{u} \omega \\
& -2 x^{T} P \tilde{B} H_{m} \omega+x^{T} C^{T} C x-\gamma^{2} \omega^{T} \omega .
\end{aligned}
$$


Using Lemma 2, we get

$$
\begin{aligned}
2 x^{T} P \Delta A_{u} x \leq & \epsilon_{1} x^{T} P^{2} x+\epsilon_{1}^{-1} x^{T} \Delta A_{u} x \\
\leq & x^{T}\left[\epsilon_{1} P^{2}+a_{u}^{2} \epsilon_{1}^{-1} I\right] x \\
-2 x^{T} P \tilde{B} \Delta A_{m} x \leq & \epsilon_{2} x^{T} P^{2} x \\
& +\epsilon_{2}^{-1} x^{T} \Delta A_{m}^{T} \tilde{B}^{T} \tilde{B} \Delta A_{m} x \\
\leq & x^{T}\left[\epsilon_{2} P^{2}+\frac{b_{u}^{2} a_{m}^{2}}{\left(1-b_{m}\right)^{2}} \epsilon_{2}^{-1} I\right] x, \\
2 x^{T} P f_{u} \leq & \epsilon_{3} x^{T} P^{2} x+\epsilon_{3}^{-1} f_{u}^{T} f_{u} \\
\leq & x^{T}\left[\epsilon_{3} P^{2}+g_{u}^{2} \epsilon_{3}^{-1} I\right] x \\
-2 x^{T} P \tilde{B} f_{m} \leq & \epsilon_{4} x^{T} P^{2} x+\epsilon_{4}^{-1} f_{m}^{T} \tilde{B}^{T} \tilde{B} f_{m} \\
\leq & x^{T}\left[\epsilon_{4} P^{2}+\frac{b_{u}^{2} g_{m}^{2}}{\left(1-b_{m}\right)^{2}} \epsilon_{4}^{-1} I\right] x, \\
2 x^{T} P \tilde{B} K x \leq & \epsilon_{5} x^{T} P^{2} x+\epsilon_{5}^{-1} x^{T} K^{T} \tilde{B}^{T} \tilde{B} K x \\
\leq & x^{T}\left[\epsilon_{5} P^{2}\right. \\
& \left.+\frac{b_{u}^{2}}{\left(1-b_{m}\right)^{2}} \epsilon_{5}^{-1} K^{T} K\right] x, \\
-2 x^{T} P \tilde{B} H_{m} \omega \leq & \epsilon_{6} x^{T} P^{2} x+\epsilon_{6}^{-1} \omega^{T} H_{m}^{T} \tilde{B}^{T} \tilde{B} H_{m} \omega \\
\leq & \epsilon_{6} x^{T} P^{2} x \\
& +\frac{b_{u}^{2}}{\left(1-b_{m}\right)^{2}} \epsilon_{6}^{-1} \omega^{T} H_{m}^{T} H_{m} \omega,
\end{aligned}
$$

Consequently, we obtain

$$
\begin{aligned}
\dot{V}+ & y^{T} y-\gamma^{2} \omega^{T} \omega \\
\leq & x^{T} \Omega x+x^{T} P H_{u} \omega+\omega^{T} H_{u}^{T} P x \\
& +\omega^{T}\left[-\gamma^{2} I+\frac{b_{u}^{2}}{\left(1-b_{m}\right)^{2}} \epsilon_{6}^{-1} H_{m}^{T} H_{m}\right] \omega,
\end{aligned}
$$

where

$$
\begin{aligned}
\Omega= & P A+A^{T} P+P B K+K^{T} B^{T} P+C^{T} C \\
& +\sum_{i=1}^{6} \epsilon_{i} P^{2}+\left(a_{u}^{2} \epsilon_{1}^{-1}+\frac{b_{u}^{2} a_{m}^{2}}{\left(1-b_{m}\right)^{2}} \epsilon_{2}^{-1}+g_{u}^{2} \epsilon_{3}^{-1}\right. \\
& \left.+\frac{b_{u}^{2} g_{m}^{2}}{\left(1-b_{m}\right)^{2}} \epsilon_{4}^{-1}\right) I+\frac{b_{u}^{2}}{\left(1-b_{m}\right)^{2}} \epsilon_{5}^{-1} K^{T} K .
\end{aligned}
$$

The inequality (25) can be reformulated as follows:

$$
\begin{aligned}
\dot{V}+ & y^{T} y-\gamma^{2} \omega^{T} \omega \\
\leq & {\left[\begin{array}{ll}
x^{T} & \omega^{T}
\end{array}\right] } \\
& \times\left[\begin{array}{cc}
\Omega & P H_{u} \\
H_{u}^{T} P & \frac{b_{u}^{2}}{\left(1-b_{m}\right)^{2}} \epsilon_{6}^{-1} H_{m}^{T} H_{m}-\gamma^{2} I
\end{array}\right]\left[\begin{array}{c}
x \\
\omega
\end{array}\right]
\end{aligned}
$$

Thus Lemma 1 is satisfied if

$$
\left[\begin{array}{cc}
\Omega & P H_{u} \\
H_{u}^{T} P & \frac{b_{u}^{2}}{\left(1-b_{m}\right)^{2}} \epsilon_{6}^{-1} H_{m}^{T} H_{m}-\gamma^{2} I
\end{array}\right]<0 .
$$

The next LMI can be derived from (26), by using Lemma 3:

$$
\left[\begin{array}{ccc}
\Omega & P H_{u} & 0 \\
H_{u}^{T} P & -\gamma^{2} I & b_{u} H_{m}^{T} \\
0 & b_{u} H_{m} & -\left(1-b_{m}\right)^{2} \epsilon_{6} I
\end{array}\right]<0 .
$$

After pre-multiplying and post-multiplying (27) by $\operatorname{diag}\left[P^{-1}, I, I\right]$, considering $X=P^{-1}$ and $R=$ $K X$, the LMI (18) is obtained by the successive use of Lemma 3. Therefore, the proof is complete.

\section{Reachability analysis}

In the preceding section a sufficient condition was derived for the quadratic stability of the uncertain system on the sliding manifold $S(t)=0$. Now, we proceed with the second task, which is the design of an SMC law, such that the reachability of the specified sliding surface is guaranteed.

4.1. Integral sliding mode control law. The proposed ISMC law is specified through the following result.

Theorem 2. Consider the uncertain system (1) with the assumptions (i)-(iv). Suppose that the switching surface is given by (6) with $K=R X^{-1}$, where $X$ and $R$ are solutions of the LMI (18). Suppose also that the SMC law is

$$
u=K x-\rho \frac{S}{\|S\|},
$$

where

$$
\begin{aligned}
\rho & =\frac{1}{1-b_{m}} \rho_{1}, \\
\rho_{1} & =q+\left(a_{m}+b_{m}\|K\|+g_{m}\right)\|x\|+\left\|H_{m}\right\| \omega_{0},
\end{aligned}
$$

with $q$ being a small positive scalar. Then a stable sliding mode exists from the initial time.

Proof. Consider the Lyapunov function

$$
V=\frac{1}{2} S^{T} S
$$

Using (13), the derivative of this function with respect to time is given as follows:

$$
\begin{aligned}
S^{T} \dot{S}= & S^{T}\left[\Delta A_{m} x+\left(I_{m}+\Delta B_{m}\right) u\right. \\
& \left.+H_{m} \omega+f_{m}-K x\right] \\
= & S^{T}\left(\Delta A_{m} x+\Delta B_{m} K x+H_{m} \omega+f_{m}\right. \\
& \left.-\left(I_{m}+\Delta B_{m}\right) \rho \frac{S}{\|S\|}\right) \\
= & S^{T}\left(\Delta A_{m} x+\Delta B_{m} K x+H_{m} \omega+f_{m}\right) \\
& -\rho\|S\|-\rho S^{T} \Delta B_{m} \frac{S}{\|S\|} \\
= & S^{T}\left(\Delta A_{m} x+\Delta B_{m} K x+H_{m} \omega+f_{m}\right) \\
& -\rho_{1}\|S\|-\frac{b_{m}}{1-b_{m}} \rho_{1} \| S \mid-\rho S^{T} \Delta B_{m} \frac{S}{\|S\|}
\end{aligned}
$$




$$
\begin{aligned}
\leq & \|S\|\left[\left\|\Delta A_{m} x\right\|+\left\|\Delta B_{m} K x\right\|+\left\|H_{m} \omega\right\|\right. \\
& \left.+\left\|f_{m}\right\|\right]-\rho_{1}\|S\|-b_{m} \rho\|S\| \\
\leq & -q\|S\|<0 .
\end{aligned}
$$

Then, the SMC law considered guarantees the reachability of the switching surface. In addition, the initial value of $S(t)$ is given by $S(0)=0$. Thus, the proof is complete.

Remark 2. The switching gain (29) of the proposed controller is a function of the norm-bounds of the matched components of uncertainties and disturbances $a_{m}, b_{m}, g_{m}$ and $\left\|H_{m}\right\|$. Moreover, $a_{m} \leq a, b_{m} \leq b, g_{m} \leq g$, $\left\|H_{m}\right\| \leq\|H\|$, which allows the optimization of the discontinuous controller magnitude. This argument justifies the procedure proposed in (11)-(12).

4.2. Adaptive fuzzy ISMC law. Two major problems may affect the applicability of the proposed SMC law. The first is the difficulty to obtain the exact values of uncertainties and disturbance bounds. The second is the phenomenon of chattering, a major disadvantage of SMC, which is induced by the switching nature of the controller. Hence, to overcome these problems, an Adaptive Fuzzy ISMC (AFISMC) law is presented in this section. The proposed AFISMC is based on the introduction of a Fuzzy Logic (FL) inference mechanism which replaces the switching control law. The switching function (6) can be written as

$$
S=\left[s_{1} \cdots s_{i} \cdots s_{m}\right]^{T} .
$$

Let $s_{i}$ be the input linguistic variable of FL, and $u_{F, i}$ be the output linguistic variable. The associated fuzzy sets are expressed as follows:

- for the antecedent proposition $\left(s_{i}\right)$ : P (Positive), $\mathrm{N}$ (Negative), and Z (Zero);

- for the consequent proposition $\left(u_{F, i}\right)$ : PE (Positive Effort), NE (Negative Effort), and ZE (Zero Effort).

In order to make the sliding surface attractive, the fuzzy linguistic rule base can be given as follows:

1. Rule 1: If $s_{i}$ is $\mathrm{P}$, then $u_{F, i}$ is PE.

2. Rule 2: If $s_{i}$ is Z, then $u_{F, i}$ is ZE.

3. Rule 3: If $s_{i}$ is $\mathrm{N}$, then $u_{F, i}$ is NE.

The membership functions of the input fuzzy sets are of the triangle type, and those of the output fuzzy sets are of the singleton type. The singleton defuzzification method is used in this work. Then the fuzzy controller (output of the defuzzification module) can be written as

$$
u_{F, i}=\frac{\sum_{k=1}^{3} \mu_{j k} \delta_{j k}}{\sum_{k=1}^{3} \mu_{j k}},
$$

where $0 \leq \mu_{j k} \leq 1$ is the firing strength of rule $k, k=$ $1, \ldots, 3, \delta_{j 1}=\delta_{j}, \delta_{j 2}=0$, and $\delta_{j 3}=-\delta_{j}$ stand for the centres of the membership functions PE, ZE, and NE, respectively. Owing to the special choice of triangular membership functions, we get

$$
\sum_{k=1}^{3} \mu_{j k}=1 .
$$

As a result, (31) can be reduced to the following:

$$
u_{F, j}=\left(\mu_{j 1}-\mu_{j 3}\right) \delta_{j} .
$$

According to the aforementioned fuzzy rule base, it is easy to observe that

$$
u_{F, j}=\left\{\begin{array}{lll}
\mu_{j 1} \delta_{j}, & \text { if } \quad s_{j}>0, \\
-\mu_{j 3} \delta_{j}, & \text { if } & s_{j}<0 .
\end{array}\right.
$$

Then we can conclude that

$$
s_{j}\left(\mu_{j 1}-\mu_{j 3}\right) \delta_{j} \geq 0 .
$$

Consider again the Lyapunov candidate function (30). As was mentioned at the beginning of the proof of Theorem 2, its derivative with respect to time is given by

$$
\begin{aligned}
\dot{V}= & S^{T}\left[\Delta A_{m} x+\left(I_{m}+\Delta B_{m}\right) u\right. \\
& \left.+H_{m} \omega+f_{m}-K x\right] .
\end{aligned}
$$

Consequently, if the controller is selected as follows:

$$
u=K x+u_{F},
$$

where $u_{F}$ is the fuzzy controller specified by

$$
u_{F}=-\frac{1}{1-b_{m}}\left[u_{F, 1} \cdots u_{F, m}\right]^{T},
$$

then (36) can be written as

$$
\dot{V}=S^{T}\left[\varphi(x, \omega, t)+\left(I_{m}+\Delta B_{m}\right) u_{F}\right],
$$

where

$$
\begin{aligned}
\varphi(x, \omega, t) & =\Delta A_{m} x+\Delta B_{m} K x+H_{m} \omega+f_{m} \\
& =\left[\varphi_{1} \cdots \varphi_{m}\right]^{T} .
\end{aligned}
$$

Therefore,

$$
\begin{aligned}
\dot{V}= & \sum_{j=1}^{m} s_{j} \varphi_{j}-\frac{1}{1-b_{m}} \sum_{j=1}^{m} s_{j} u_{F, j}-S^{T} \Delta B_{m} u_{F} \\
\leq & \sum_{j=1}^{m}\left|s_{j} \varphi_{j}\right|-\frac{1}{1-b_{m}} \sum_{j=1}^{m} s_{j}\left(\mu_{j 1}-\mu_{j 3}\right) \delta_{j} \\
& +\left\|\Delta B_{m}\right\|\left\|S^{T} u_{F}\right\| \\
\leq & \sum_{j=1}^{m}\left|s_{j} \varphi_{j}\right|-\frac{1}{1-b_{m}} \sum_{j=1}^{m} s_{j}\left(\mu_{j 1}-\mu_{j 3}\right) \delta_{j} \\
& +\frac{b_{m}}{1-b_{m}} \sum_{j=1}^{m} s_{j}\left(\mu_{j 1}-\mu_{j 3}\right) \delta_{j} .
\end{aligned}
$$




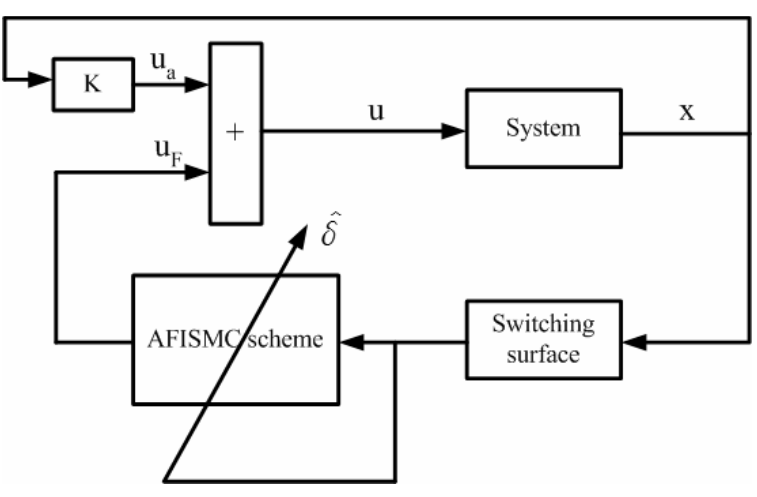

Fig. 1. Structure of the proposed AFISMC scheme.

Hence we get

$$
\dot{V} \leq \sum_{j=1}^{m}\left|s_{j}\right|\left[\left|\varphi_{j}\right|-\left|\mu_{j 1}-\mu_{j 2}\right| \delta_{j}\right] .
$$

As a result, $\dot{V}<0$ if the following inequality holds:

$$
\delta_{j}>\frac{\left|\varphi_{j}\right|}{\left|\mu_{j 1}-\mu_{j 3}\right|}, \quad j=1, \ldots, m .
$$

According to Wang's theorem (Wang, 1997), there exists an optimal value $\overline{\delta_{j}}$ which satisfies the preceding inequality. However, this value cannot be accurately determined because the uncertainties bounds cannot be easily extracted. Then, $\delta_{j}$ is chosen as the parameter to be updated. The structure of the proposed scheme is indicated in Fig. 1. The following theorem describes this control law.

Theorem 3. Consider the uncertain system (1) with the assumptions (i)-(iv). Suppose that the switching surface is given by (6), where $X$ and $R$ are solutions to be LMI (18). If the control law is given by (37), where $u_{F}$ is the fuzzy controller (38), and $\delta_{j}$ is replaced by the adaptive parameter $\hat{\delta_{j}}$ described as follows:

$$
\dot{\hat{\delta_{j}}}=\beta_{j} s_{j}\left(\mu_{j 1}-\mu_{j 3}\right),
$$

with $\beta_{j}$ being a nonnegative scalar, then a stable sliding mode exists from the initial time.

Proof. The estimated error between the adaptive parameter $\hat{\delta_{j}}$ and the optimal value $\overline{\delta_{j}}$ is defined as

$$
\tilde{\delta}_{j}=\hat{\delta}_{j}-\overline{\delta_{j}}
$$

Thereafter, we choose the following Lyapunov candidate:

$$
V_{1}=V+\frac{1}{2} \sum_{j=1}^{m} \beta_{j}^{-1} \tilde{\delta_{j}^{2}} .
$$

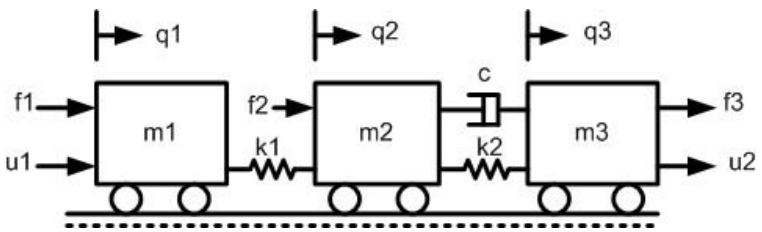

Fig. 2. Mechanical system example.

Thus we obtain

$$
\dot{V}_{1}=\dot{V}+\sum_{j=1}^{m} \beta_{j}^{-1} \tilde{\delta}_{j} \dot{\tilde{\delta}}_{j}
$$

By using (37), (39), (43) and (44), (46) can be rewritten as

$$
\begin{aligned}
& \dot{V}_{1}=\sum_{j=1}^{m} s_{j} \varphi_{j}-\frac{1}{1-b_{m}} \sum_{j=1}^{m} s_{j} u_{F, j}-S^{T} \Delta B_{m} u_{F} \\
& +\sum_{j=1}^{m}\left(\tilde{\delta_{j}}-\overline{\delta_{j}}\right) s_{j}\left(\mu_{j 1}-\mu_{j 3}\right) \\
& =\sum_{j=1}^{m}\left[s_{j} \phi_{j}-s_{j} \overline{\delta_{j}}\left(\mu_{j 1}-\mu_{j 3}\right)\right]-S^{T} \Delta B_{m} u_{F} \\
& -\frac{1}{1-b_{m}} \sum_{j=1}^{m} s_{j} u_{F, j}+\sum_{j=1}^{m} \hat{\delta}_{j} s_{j}\left(\mu_{j 1}-\mu_{j 2}\right) \\
& \leq-\frac{1}{1-b_{m}} \sum_{j=1}^{m} s_{j} \hat{\delta}_{j}\left(\mu_{j 1}-\mu_{j 3}\right) \\
& +\sum_{j=1}^{m} s_{j} \hat{\delta}_{j}\left(\mu_{j 1}-\mu_{j 3}\right) \\
& +\sum_{j=1}^{m}\left[s_{j} \phi_{j}-s_{j} \overline{\delta_{j}}\left(\mu_{j 1}-\mu_{j 3}\right)\right] \\
& +\left\|\Delta B_{m}\right\|\left\|S^{T} u_{F}\right\| \\
& \leq \sum_{j=1}^{m}\left|s_{j}\right|\left|\phi_{j}\right|-\overline{\delta_{j}}\left|\mu_{j 1}-\mu_{j 3}\right| \\
& -\sum_{j=1}^{m} s_{j} \hat{\delta}_{j}\left(\mu_{j 1}-\mu_{j 3}\right) \\
& +\sum_{j=1}^{m} s_{j} \hat{\delta}_{j}\left(\mu_{j 1}-\mu_{j 3}\right) \\
& \leq \sum_{j=1}^{m} \mid s_{j}\left[|| \phi_{j}\left|-\overline{\delta_{j}}\right| \mu_{j 1}-\mu_{j 3} \mid\right]<0 .
\end{aligned}
$$

Thus, the time derivative of the Lyapunov candidate function is negative. This completes the proof.

\section{Example}

In this section we shall evaluate the proposed control laws through the application to a sixth-order mechanical sys- 


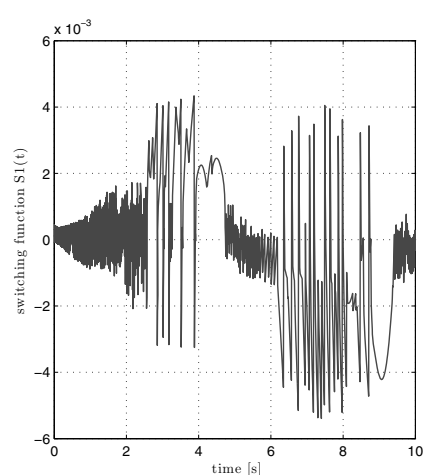

(a)

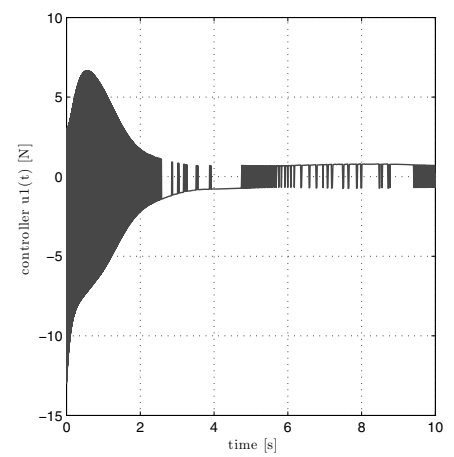

(c)

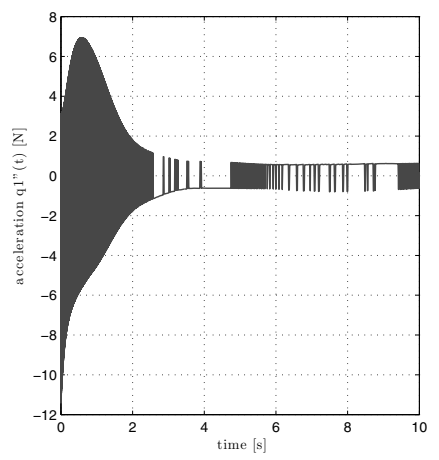

(e)

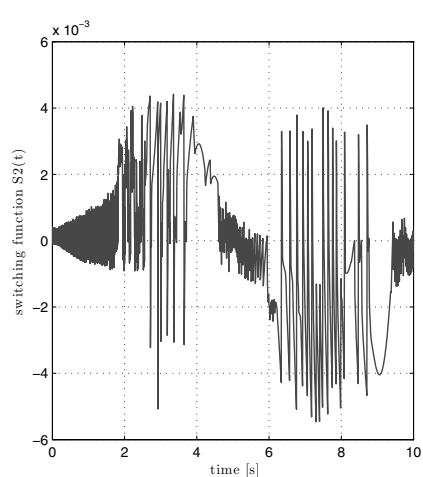

(b)

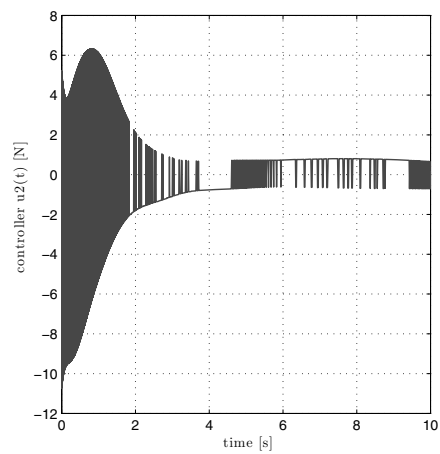

(d)

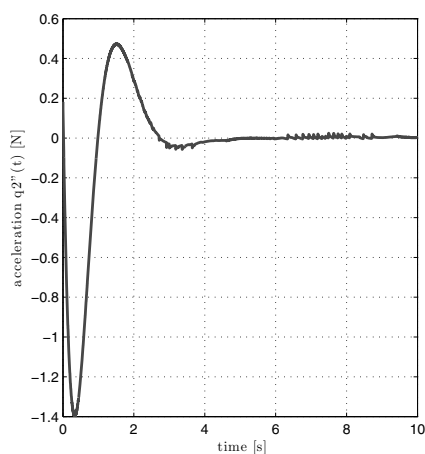

(f)

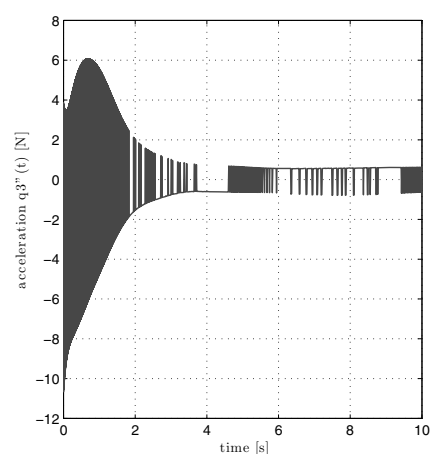

(g)

Fig. 3. Simulation results using ISMC: switching function $S_{1}(t)$ (a), switching function $S_{2}(t)$ (b), controller $u_{1}(t)$ (c), controller $u_{2}(t)$ (d), acceleration $\ddot{q}_{1}(t)(\mathrm{e})$, acceleration $\ddot{q}_{2}(t)(\mathrm{f})$, acceleration $\ddot{q}_{3}(t)(\mathrm{g})$. 


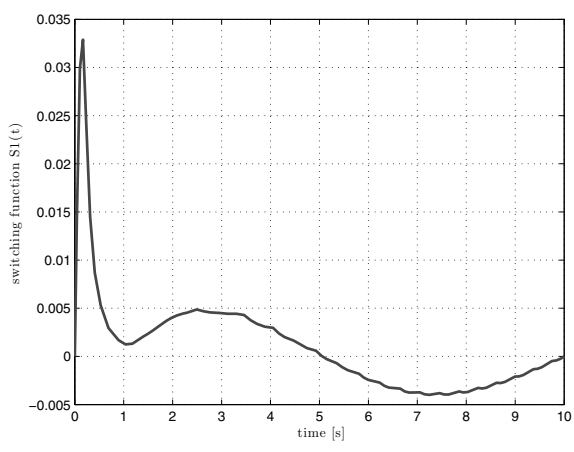

(a)

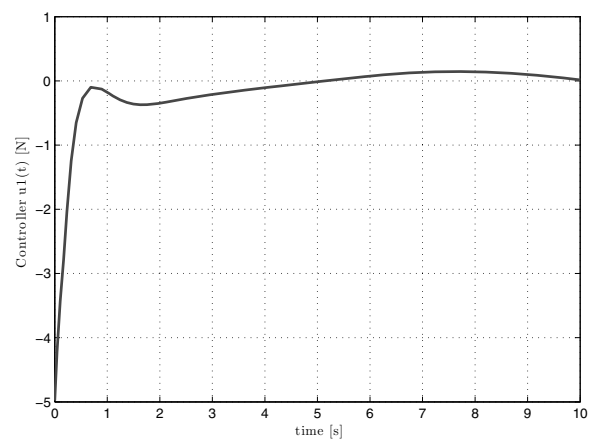

(c)

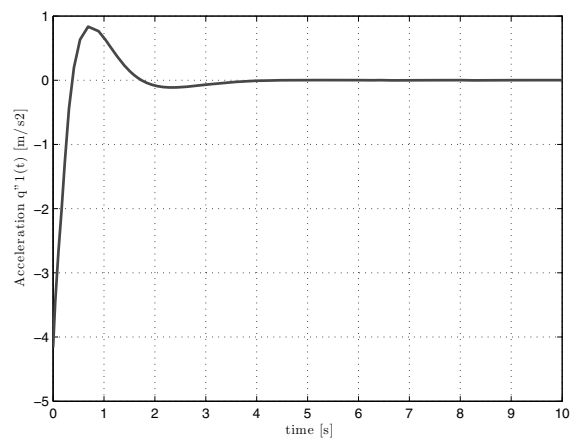

(e)

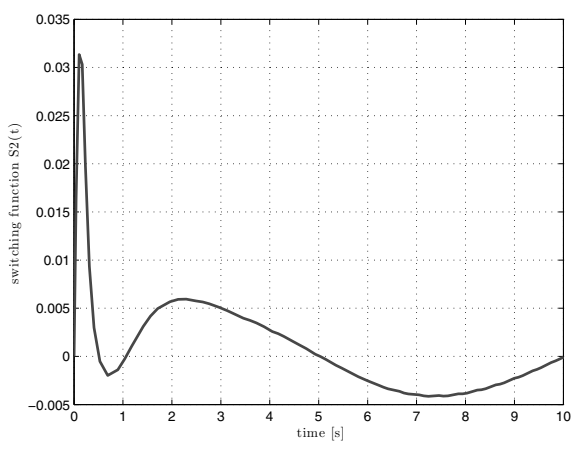

(b)

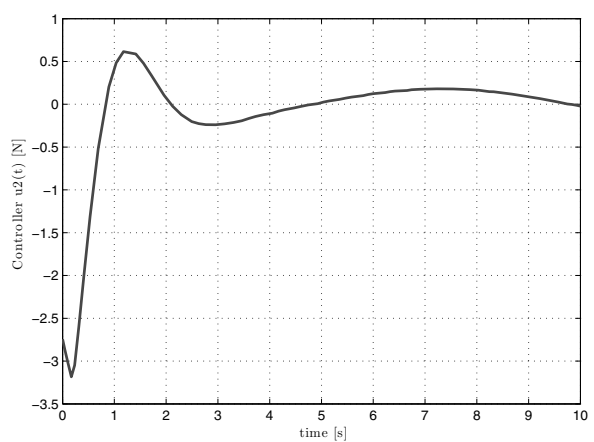

(d)

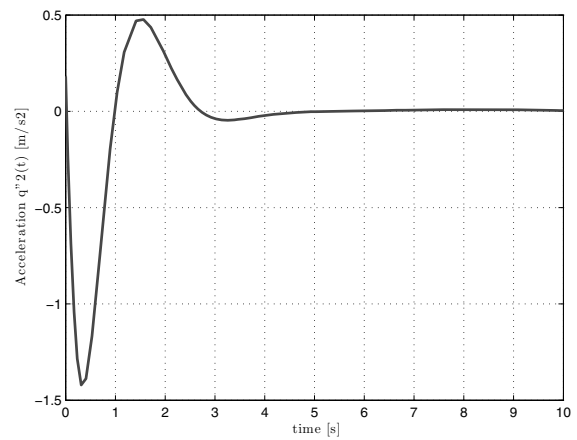

(f)

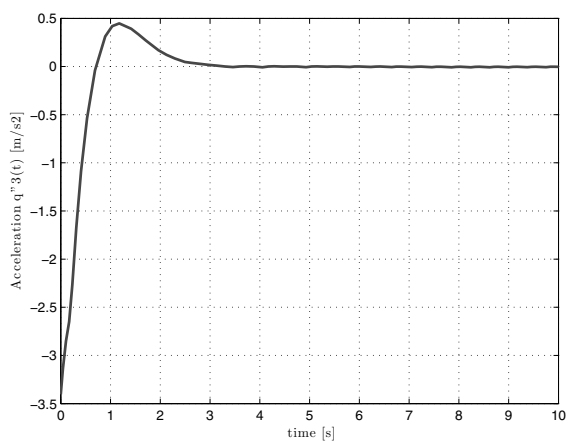

(g)

Fig. 4. Simulation results using AFISMC: switching function $S_{1}(t)$ (a), switching function $S_{2}(t)$ (b), controller $u_{1}(t)$ (c), controller $u_{2}(t)(\mathrm{d})$, acceleration $\ddot{q}_{1}(t)(\mathrm{e})$, acceleration $\ddot{q}_{2}(t)(\mathrm{f})$, acceleration $\ddot{q}_{3}(t)(\mathrm{g})$. 
tem shown in Fig. 2. Suppose that

$$
\begin{aligned}
m_{i} & =m+\Delta m_{i} \\
& =m\left(1+\xi_{i}\right), \quad i=1, \ldots, 3, \quad m=1, \\
k_{1} & =k_{2}=2, \quad c=3, \\
x & =\left[\begin{array}{llllll}
q_{1} & \dot{q_{1}} & q_{2} & \dot{q_{2}} & q_{3} & \dot{q_{3}}
\end{array}\right]^{T}, \\
u & =\left[\begin{array}{lll}
u_{1} & u_{2}
\end{array}\right]^{T}, \quad \omega=\left[\begin{array}{lll}
f_{1} & f_{2} & f_{3}
\end{array}\right]^{T} .
\end{aligned}
$$

The system can be described by the following state equation:

$$
\dot{x}=(A+\Delta A) x+(B+\Delta B) u+H \omega,
$$

with

$$
\begin{aligned}
& A=\left[\begin{array}{cccccc}
0 & 1 & 0 & 0 & 0 & 0 \\
-2 & 0 & 2 & 0 & 0 & 0 \\
0 & 0 & 0 & 1 & 0 & 0 \\
2 & 0 & -4 & -3 & 2 & 3 \\
0 & 0 & 0 & 0 & 0 & 1 \\
0 & 0 & 2 & 3 & -2 & -3
\end{array}\right] \\
& B=\left[\begin{array}{ll}
0 & 0 \\
1 & 0 \\
0 & 0 \\
0 & 0 \\
0 & 0 \\
0 & 1
\end{array}\right], \quad H=\left[\begin{array}{lll}
0 & 0 & 0 \\
1 & 0 & 0 \\
0 & 0 & 0 \\
0 & 1 & 0 \\
0 & 0 & 0 \\
0 & 0 & 1
\end{array}\right] \\
& \Delta A=\left[\begin{array}{cccccc}
0 & 0 & 0 & 0 & 0 & 0 \\
2 \zeta_{1} & 0 & -2 \zeta_{1} & 0 & 0 & 0 \\
0 & 0 & 0 & 0 & 0 & 0 \\
-2 \zeta_{2} & 0 & 4 \zeta_{2} & 3 \zeta_{2} & -2 \zeta_{2} & -3 \zeta_{2} \\
0 & 0 & 0 & 0 & 0 & 1 \\
0 & 0 & -2 \zeta_{3} & -3 \zeta_{3} & 2 \zeta_{3} & 3 \zeta_{3}
\end{array}\right] \text {, } \\
& \Delta B=\left[\begin{array}{cc}
0 & 0 \\
-\zeta_{1} & 0 \\
0 & 0 \\
0 & 0 \\
0 & 0 \\
0 & -\zeta_{3}
\end{array}\right] \\
& \zeta_{i}=\frac{\xi_{i}}{1+\xi_{i}}, \quad f_{i}=0.1 \sin (\pi t) \text {. }
\end{aligned}
$$

For $\xi_{i}=0.1, i=1, \ldots, 3$, applying the decomposition procedure given by (11)-(12), we get $a_{m}=$ $0.4711, a_{u}=0.5892, b_{m}=0.0909, b_{u}=0$. Then a feasible solution of the LMI (18) is given by

$$
X=\left[\begin{array}{ccc}
0.055 & -0.099 & -0.005 \\
-0.099 & 0.380 & 0.015 \\
-0.005 & 0.015 & 0.062 \\
0.023 & -0.072 & -0.070 \\
-0.015 & -0.001 & -0.010 \\
0.027 & -0.015 & 0.012
\end{array}\right.
$$

$$
\begin{aligned}
& \begin{array}{lll}
0.023 & -0.015 & 0.027
\end{array} \\
& \begin{array}{lll}
-0.072 & -0.001 & -0.015
\end{array} \\
& \begin{array}{lll}
-0.070 & -0.010 & 0.012
\end{array} \\
& \begin{array}{lll}
0.385 & 0.038 & -0.129
\end{array} \\
& \begin{array}{lll}
0.038 & 0.053 & -0.075
\end{array} \\
& \begin{array}{lll}
-0.129 & -0.075 & 0.238
\end{array} \\
& \epsilon_{1}=0.0385, \quad \gamma=1.4534 \text {, } \\
& K=\left[\begin{array}{ccc}
-2.661 & -3.862 & -2.453 \\
2.285 & 0.922 & -3.025
\end{array}\right. \\
& \left.\begin{array}{ccc}
0.286 & -4.266 & -1.972 \\
-3.742 & -0.740 & -0.637
\end{array}\right]
\end{aligned}
$$

We remark that none of the traditional design methods of Kim et al. (2000), Choi (2001) or Xia and Jia (2003) nor the integral SMC methods given by Cao and Xu (2004), Shaocheng and Yongji (2006) or Choi (2007) is applicable to the example considered. The simulation results are obtained for the initial state vector

$$
x(0)=\left[\begin{array}{llllll}
0.1 & 0.2 & 0.3 & 0.4 & 0.5 & 0.6
\end{array}\right]^{T} .
$$

Figures 3 and 4 indicate the evolution of switching function components, controller components, and accelerations using the proposed ISMC (28) and AFISMC (37)-(43), respectively. The displacement evolution for both ISMC and AFISMC methods is shown in Fig. 5. From these simulation results it is clear that the proposed schemes result in a stable sliding mode from the initial time. However, it is obvious from the controller evolution that the first approach is accompanied with the chattering phenomenon, which induces the appearance of an undesirable vibration as depicted by the evolution of accelerations. Fortunately, this disadvantage is overcome by AFISMC through the elimination of high frequency discontinuities in both the controller and acceleration. In addition, Fig. 5 shows that the last approach preserves the same dynamical performances of the closed loop system as the first one.

Figure 6 shows the displacement evolution for both the nominal and uncertain systems. The displacements $q_{1}(t)$ and $q_{3}(t)$, which are directly actuated by the control input, are superposed for both systems, while there exist a slight difference in the case of $q_{2}(t)$ representing a nonactuated variable. Therefore the robustness of the proposed approach is confirmed.

\section{Conclusion}

A robust ISMC design for mismatched uncertain systems has been studied. A sufficient condition for quadratic stability of sliding motion has been established in terms of LMIs. The immediate sliding mode existence has been guaranteed by the proposed ISMC law. The induced chattering phenomenon has been eliminated by the introduction of an AFISMC law. Finally, the effectiveness of the 


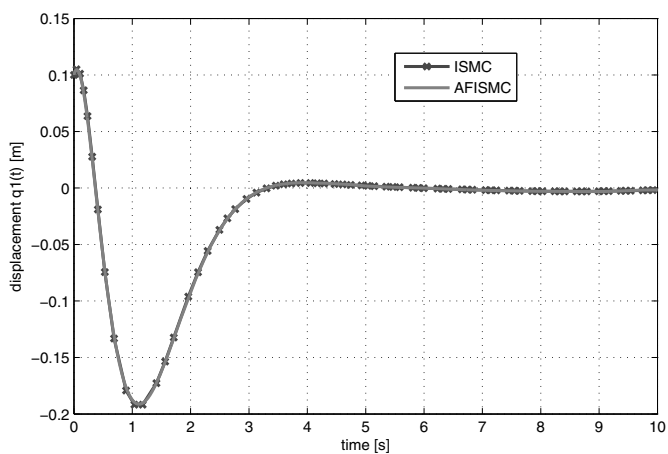

(a)

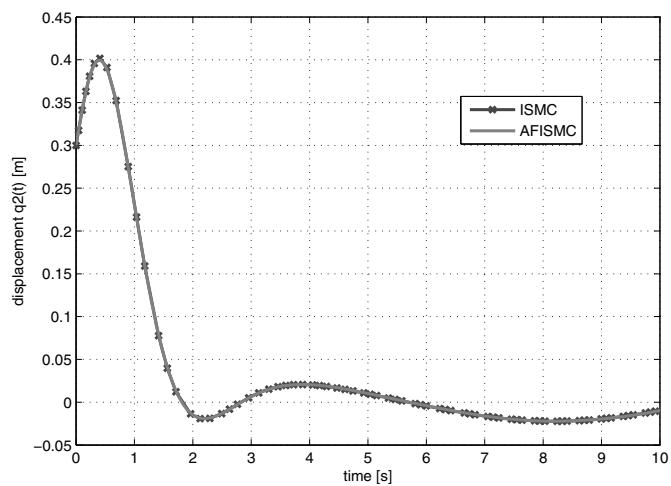

(b)

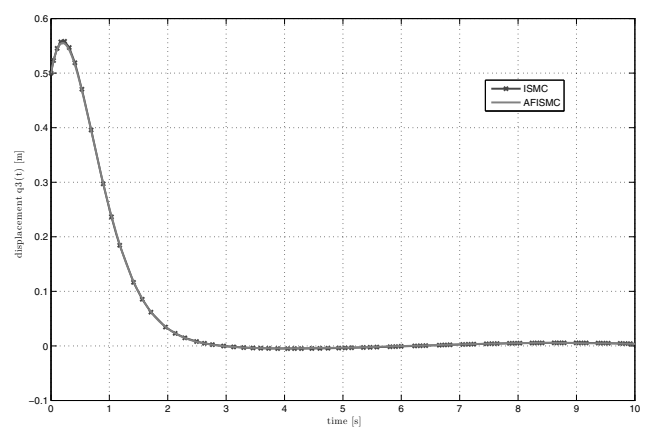

(c)

Fig. 5. Displacement evolution according to both ISMC and AFISMC: displacement $q_{1}(t)$ (a), displacement $q_{2}(t)$ (b), displacement $q_{3}(t)$ (c).

proposed methods has been proved through a sixth order uncertain mechanical system example.

\section{References}

Ackermann, J. and Utkin, V.I. (1998). Sliding mode control design based on Ackermann's formula, IEEE Transactions on Automatic Control 43(2): 234-237.

Boyd, S., Ghaoui, L.E., Feron, E. and Balakrishnan, V. (1994). Linear Matrix Inequalities in System and Control Theory,

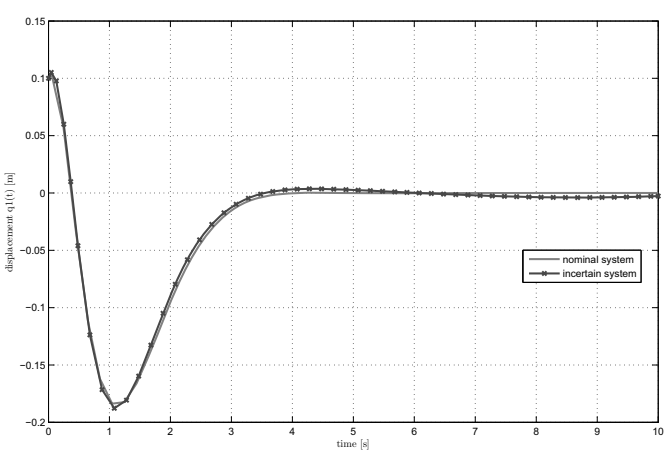

(a)

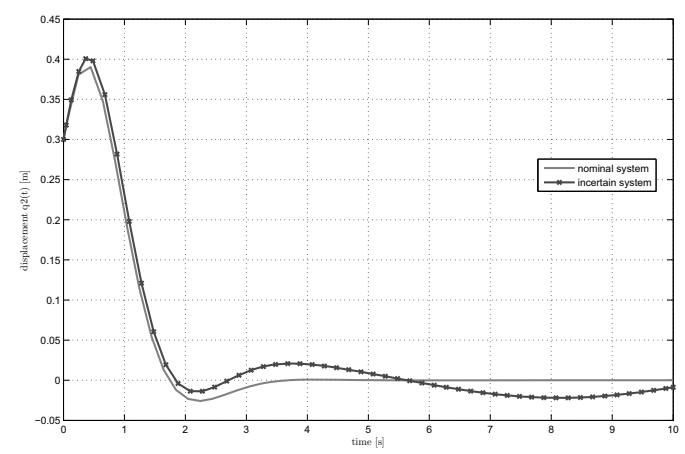

(b)

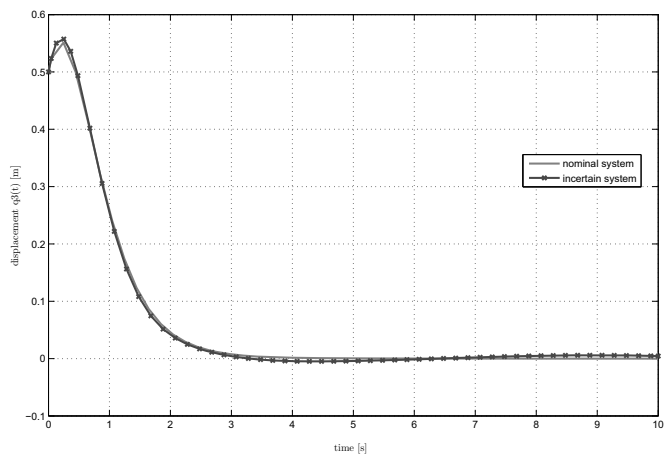

(c)

Fig. 6. Displacement evolution for the nominal and uncertain system according to AFISMC: displacement $q_{1}(t)$ (a), displacement $q_{2}(t)$ (b), displacement $q_{3}(t)$ (c).

Society for Industrial and Applied Mathematics, Philadelphia, PA.

Cao, W.J. and Xu, J.X. (2004). Nonlinear integral-type sliding surface for both matched and unmatched uncertain systems, IEEE Transactions on Automatic Control 49(8): 1355-1360.

Choi, H.H. (2001). Variable structure output feedback control design for a class of uncertain dynamic systems, Automatica 38(2): 335-341.

Choi, H.H. (2007). LMI-based sliding surface design for integral sliding mode control of mismatched uncertain systems, 
IEEE Transactions on Automatic Control 52(4): 736-742.

Decarlo, R.A., Zak, S.H. and Matthews, G.P. (1988). Variable structure control of nonlinear multivariable systems: A tutorial, Proceeding of IEEE 76(3): 212-232.

Ha, Q.P., Rye, D.C. and Durrant-Whyte, H.F. (1999). Robust sliding mode control with application, International Journal of Control 72(12): 1078-1096.

Hung, J.Y., Gao, W.B. and Hung, J.C. (1993). Variable structure control: A survey, IEEE Transactions on Industrial Electronics 40(1): 2-22.

Kim, S., Park, Y. and Oh, S.H. (2000). Designing robust sliding hyperplanes for parametric uncertain systems: A Riccati approach, Automatica 36(7): 1041-1048.

Mnasri, C. and Gasmi, M. (2007a). Decentralized sliding mode control for linear interconnected systems, Proceedings of the 4th International Multi-Conference on Systems Signals and Devices, SSD 2007, Hammamet, Tunisia.

Mnasri, C. and Gasmi, M. (2007b). Robust decentralized sliding mode control for large scale uncertain systems, Proceedings of the 26th Chinese Control Conference, CCC 2007, Hunan, China, pp. 76-81.

Mnasri, C. and Gasmi, M. (2008). Adaptive fuzzy sliding mode model-reference control for mimo uncertain systems, International Review of Automatic Control, IREACO 01(2): 143-152.

Shaocheng, Q. and Yongji, W. (2006). Robust control of uncertain time delay system: A novel sliding mode control design via LMI, Journal of Systems Engineering and Electronics 17(3): 624-628.

Utkin, V.I. (1992). Sliding Modes in Control and Optimization, Springer-Verlag, Berlin.

Utkin, V.I. and Shi, J. (1996). Integral sliding mode in systems operating under uncertainty conditions, Proceedings of the 35th Conference on Decision and Control, Kobe, Japan, pp. 4591-4596.
Wang, L.X. (1997). A Course in Fuzzy Systems and Control, Prentice Hall, Englewood Cliffs, NJ.

Xia, Y. and Jia, Y. (2003). Robust sliding mode control design of uncertain time-delay systems: An LMI approach, IEEE Transactions on Automatic Control 48(6): 624-628.

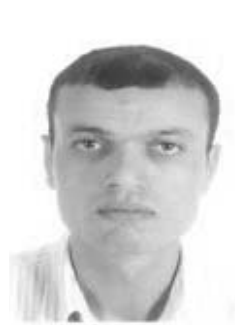

Chaouki Mnasri was born in Kasserine, Tunisia, in 1977. He received the engineering diploma in electrical engineering in 2000, the Master's degree in automatic control and signal processing in 2004, and the Ph.D. degree in electrical engineering in 2009, all from the Tunis National School of Engineering (ENIT). Now he is an associate professor in the Department of Electronics at the High Institute of Applied Sciences and Technology of Mateur (ISSAT Mateur), Tunisia. His domain of interests is related to the field of sliding mode control of MIMO systems and robust control of uncertain systems.

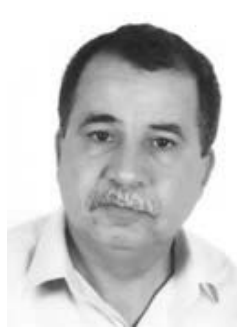

Moncef Gasmi was born in Tunis, Tunisia, in 1958. He received the principal engineering diploma in electrical engineering in 1984, the Master's degree in systems analysis and computational treatment in 1985 , a doctorate in automatic control in 1989 and a state doctorate in electrical engineering in 2001, all from the Tunis National School of Engineering (ENIT). Now, he is a professor and the director of the Research Unit in Automatic Control and Industrial Computing (URAII) at the National Institute of Applied Sciences and Technology (INSAT). His domain of interests is related to the modelling, analysis and control of complex systems.

Received: 1 June 2010

Revised: 21 March 2011

Re-revised: 11 June 2011 